\title{
CONTROLLED SYMMETRIES AND PASSIVE WALKING
}

\author{
Mark W. Spong and Francesco Bullo* \\ * Coordinated Science Laboratory, University of Illinois, $1308 \mathrm{~W}$. \\ Main St. Urbana, IL 61801
}

\begin{abstract}
It was shown in Spong [1999b] that the passive gaits for a planar 2-DOF biped walking on a shallow slope can be made slope invariant by a passivity based control that compensates only the gravitational torques acting on the biped. In this paper we extend these results to the general case of a $3-\mathrm{D}, n$-DOF robot. We show that if there exists a passive walking gait, i.e. a stable limit cycle, then there exists a passivity-based nonlinear control law that renders the limit cycle slope invariant. The result is constructive in the sense that we generate the resulting control law and initial conditions from the initial conditions of the passive biped and the ground slope. This intuitively simple result relies on some well-known symmetries in the dynamics of mechanical systems with respect to the group action of $\mathrm{SO}(3)$ on solution trajectories of the system. We also discuss the design of an additional passivity based control term designed to increase the basin of attraction of the passive limit cycle.
\end{abstract}

Keywords: Bipedal Locomotion, Passive Walking, Symmetry, Invariance, Limit Cycle, Nonlinear Control, Group Action, Passivity.

\section{INTRODUCTION}

The notion that it is possible to achieve walking gaits from mechanical bipeds powered only by gravity has intriqued researchers since the pioneering work of McGeer more than a decade ago (McGeer [1990]). These so-called passive gaits may help to explain the efficiency of human and animal locomotion and provide insight into the development of walking robots. For example, in human walking, there is evidence that a great part of the swing phase is passive - the muscles of the human leg are active primarily during the double support period, after which they essentially turn off and allow the leg to swing through like a jointed pendulum McMahon [1984].

Several researchers have studied passive walking in planar mechanisms, with and without knees and analyzed their passive gaits (Collins et al. [2001], Garcia et al. [1998], Goswami et al. [1997, 1998], McGeer [1990]). The stable passive gaits found in these mechanisms typically exist only for very shallow slopes and exhibit extreme sensitivity to slope magnitude.

The first results in active feedback control in this context appeared in Goswami et al. [1997] and Spong [1999a,b]. In Spong [1999a,b] it was shown, for a fully actuated compass gait biped, that the passive limit cycle of Goswami et al. [1997] can be made slope invariant using a so-called passivity based nonlinear control. Related results appear in Ohta et al. [1999] and Suzuki et al. [2001].

Passive walking in three-dimensions was investigated in Kuo [1999]. Passive limit cycles were found in the lateral plane as well as the saggital plane. However, the lateral motion was unstable and had to be compensated by feedback control. Kuo used an elegant control algorithm to adjust the foot placement at each step to achieve stable locomotion in both the lateral and sagittal planes. More recently, true three dimensional pas- 
sive walking has been achieved in Collins et al. [2001]. This remarkable biped has both knees and specially shaped feet to stabilize the lateral motion passively, arms that swing coupled to the leg motion to stabilize yaw motion and produces surprisingly anthropomorphic motion without actuation of any kind.

Motivated in part by the above work showing that passive walking can be achieved in three dimensions, we extend here our previous results on passivity based nonlinear control to the general case of a three dimensional $n$-DOF biped. The result follows nearly identically to the $2-\mathrm{D}$ case as a consequence of some symmetry properties in the Euler-Lagrange equations describing the biped. Specifically, we show that changing the ground slope defines a group action on the configuration manifold of the system and that both the kinetic energy and impact dynamics are invariant under this group action. Hence, to achieve invariance of the passive limit cycles, one need only compensate the potential energy as in the planar, 2-D case.

We also investigate an additional passivity based design in order to enlarge the basin of attraction of the system. We add to the gravity compensating control an additional term proportional to the difference of the total energy of the robot and the energy along the limit cycle. We show via simulation that this term increases the basin of attraction of the limit cycle.

\section{BACKGROUND}

We consider a general $n$-degree-of-freedom biped in 3-dimensions. The act of walking involves both

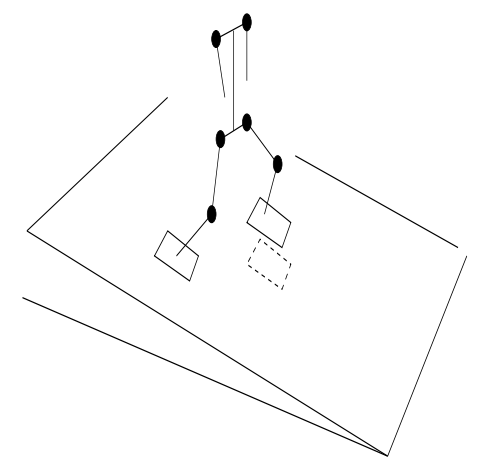

Fig. 1. A General 3-D Biped

a swing phase and a stance phase for each leg as well as impacts between the swing leg and ground. We make the standard assumptions, namely,

(i) impacts are perfectly inelastic (no bounce),

(ii) transfer of support between swing and stance legs is instantaneous,

(iii) there is no slipping at the stance leg ground contact.
Under these assumptions it can be shown (Hurmuzlu and Moskowitz [1986]) that each impact results in an instantaneous jump in velocities, hence a discontinuity in kinetic energy. The position variables are continuous through the impact and so, if the kinetic energy dissipated during the impact is somehow compensated so that the joint angles and velocities after impact are restored to their original values at the beginning of the step, then a periodic gait (limit cycle) results. In passive walking this is achieved by starting the biped on a constant downhill slope so that that loss of kinetic energy is compensated by the change in potential energy during the step. The loss of kinetic energy can also be compensated by active control of actuators at the joints so that walking can be achieved on level ground and/or uphill.

\subsection{Group Actions and Invariance}

\section{Group Actions}

We now give some background from differential geometry and dynamical systems theory (see Olver [1993]).

Definition 2.1. Let $Q$ be a differentiable manifold and $G$ be a Lie group. Then $G$ is said to act on $Q$ if there is a mapping $\Phi: G \times Q \rightarrow Q$ taking a pair $(g, q)$ to $\Phi(g, q)=\Phi_{g}(q) \in Q$ and satisfying for all $q \in Q$

(i) $\Phi_{e}(q)=q$, where $e$ is the identity element of $G$, and

(ii) $\Phi_{g_{1}}\left(\Phi_{g_{2}}(q)\right)=\Phi_{g_{1} g_{2}}(q)$.

The mapping $\Phi$ is called a lift action.

A group action on $Q$ induces corresponding maps on scalar functions over $Q$ (e.g., the system's kinetic and potential energy), tangent vectors and vector fields (e.g., the system's instantaneous velocity), and one forms (e.g., the external forces applied to the system). For example, if $h: Q \rightarrow \Re$ is a scalar function on $Q$, then the group action induces a map via composition,

$$
(h \circ \Phi)(q):=h(\Phi(q))
$$

We say that the scalar function $h(q)$ is invariant (under the group action) if, for all $g \in G$

$$
h \circ \Phi_{g}=h .
$$

Let $T_{q} Q$ be the linear space of tangent vectors at $q$, and let $g$ be an element in $G$. Let $T_{q} \Phi_{g}$ be the tangent map to $\Phi_{g}$ mapping $T_{q} Q$ onto $T_{\Phi_{g}(q)} Q$. We call $T_{q} \Phi_{g}$ the lifted action. In other words, if $X_{q} \in T_{q} Q$ and $h$ is a function on $Q$, then

$$
\left(T_{q} \Phi_{g} \circ X_{q}\right) h=X_{q}\left(h\left(\Phi_{g}(q)\right)\right) .
$$


The vector field $X$ is invariant if, for all $g \in G$,

$$
T_{q} \Phi_{g} \circ X=X \circ \Phi_{g} .
$$

Given any curve $\eta:[0, T] \rightarrow Q$, the velocity of the curve $\Phi_{g} \circ \eta$ satisfies the equality

$$
\frac{d}{d t} \Phi_{g}(\eta(t))=T_{\eta(t)} \Phi_{g} \cdot \dot{\eta}(t) .
$$

If the group action on $Q$ is commutative, the tangent map $T_{q} \Phi_{g}$ is the identity map. If the action is not commutative, e.g., if the symmetry group is $\mathrm{SO}(3)$, the action on the velocity variables is non-trivial. In the latter case, the action is the identity map if the velocities are written in the body-fixed frame.

The importance of these definitions for us is the following.

Lemma 2.2. Let the vector field $X$ be $\Phi$-invariant, and let $\gamma:[0, T] \rightarrow Q ; t \mapsto \gamma(t)$ be an integral curve of $X$, i.e., the solution of the differential equation defined by $X$ with initial condition $\gamma(0)$. Then, $\Phi_{g} \circ \gamma:[0, T] \rightarrow Q ; t \mapsto \Phi_{g}(\gamma(t))$ is an integral curve for $X$ for all $g \in G$.

See Olver [1993]

\section{WALKING ROBOTS AND PASSIVE DYNAMIC}

Consider the $n$-link biped during a single step. We use $Q=\mathrm{SO}(3) \times S$ to represent the configuration space of the biped, where $\mathrm{SO}(3)$ is the Rotation Group in $\Re^{3}$ and $S:=T^{n-3}$ is the $n-3$-torus (with $T=[0,2 \pi)$ ). A configuration is then an ordered pair $q=(R, r)$ where $R \in \mathrm{SO}(3)$ is the orientation of the first link, and $r \in S$ is the shape of the multi-body chain, for example the angle of each joint referenced to the previous joint.

In the case of a planar mechanism $Q=\mathrm{SO}(2) \times$ $S$ and, in this case, we may identify $Q$ with $T^{n}$ since elements of $\mathrm{SO}(2)$ can be represented by scalars (angles). In the case of a serial link mechanism we may again identify $Q$ with $T^{n}$ using the familiar Denavit-Hartenberg variables to define the configuration, $q$.

The advantage of this formalism is that only the first degree of freedom is reference to an absolute or world frame. The remaining joint variables are then invariant under a change of basis of the world frame. Configuration spaces that can be written as the Cartesian product of a Lie group and a shape space are referred to as principal bundles (Spivak [1979]).

3.0.1. Lagrangian Dynamics We refer to the tangent bundle $T Q$ as the space of configuration and velocities, $(q, \dot{q}) \in T Q$. In terms of the generalized coordinates $q$ and in their time-derivatives $\dot{q}$, the kinetic energy $\mathcal{K}$, potential energy $\mathcal{V}$ (due to gravity), and Lagrangian function, $\mathcal{L}$ are defined as usual, with

$$
\mathcal{L}(q, \dot{q})=\mathcal{K}(q, \dot{q})-\mathcal{V}(q) .
$$

The Euler-Lagrange equations of a passive walker, i.e., without external control inputs, are

$$
\frac{d}{d t} \frac{\partial \mathcal{L}}{\partial \dot{q}}-\frac{\partial \mathcal{L}}{\partial q}=0
$$

\subsection{Slope Changing Symmetry}

The orientation of the ground, i.e., its slope, can be represented by a rotation of the world coordinate frame, i.e. by an element of $\mathrm{SO}(3)$. The act of changing the ground slope is thus represented by an action of $\mathrm{SO}(3)$ on $Q$, that is a map $\Phi$ from $(A, q)=(A,(R, r)) \in \mathrm{SO}(3) \times Q$ into $Q$ such that

$$
\Phi(A,(R, r))=\Phi_{A}(R, r)=(A \cdot R, r) .
$$

Next, we state the following proposition.

Proposition 3.1. The kinetic energy $\mathcal{K}$ is invariant under the slope changing action $\Phi$, i.e., for all $A \in \mathrm{SO}(3)$

$$
\mathcal{K} \circ T_{q} \Phi_{A}=\mathcal{K}
$$

In terms of the generalized coordinates, this means $\mathcal{K}(q, \dot{q})=\mathcal{K}\left(\Phi_{A}(q), T_{q} \Phi_{A}(\dot{q})\right)$.

Proof: We recall that the kinetic energy $\mathcal{K}$ of a single rigid body (cf:Figure 1) can be expressed as the sum of the translational and rotational kinetic energies as

$$
\mathcal{K}=\frac{1}{2} m \dot{P}^{T} \dot{P}+\frac{1}{2} \omega^{T} I \omega .
$$

It is easily shown that both the scalar product, $\dot{P}^{T} \dot{P}$, and triple product, $\omega^{T} I \omega$, are independent of the particular world coordinate system, i.e., are invariant under a rotation of the world frame (see Spong and Vidyasagar [1989] for the details). In the general case of an $n$-DOF biped with configuration $q=(R, r)$, only the first DOF is referenced to the world frame. Since its kinetic energy is invariant under rotations of the world frame, it follows that the kinetic energy of the entire system is invariant.

3.1.1. Impact Dynamics The impact of the swing leg with the ground induces a discontinuity in the leg velocities. Under the assumptions made above on the nature of the impacts, the change in velocity is found by integrating the Euler-Lagrange equations over the (infinitesimally 


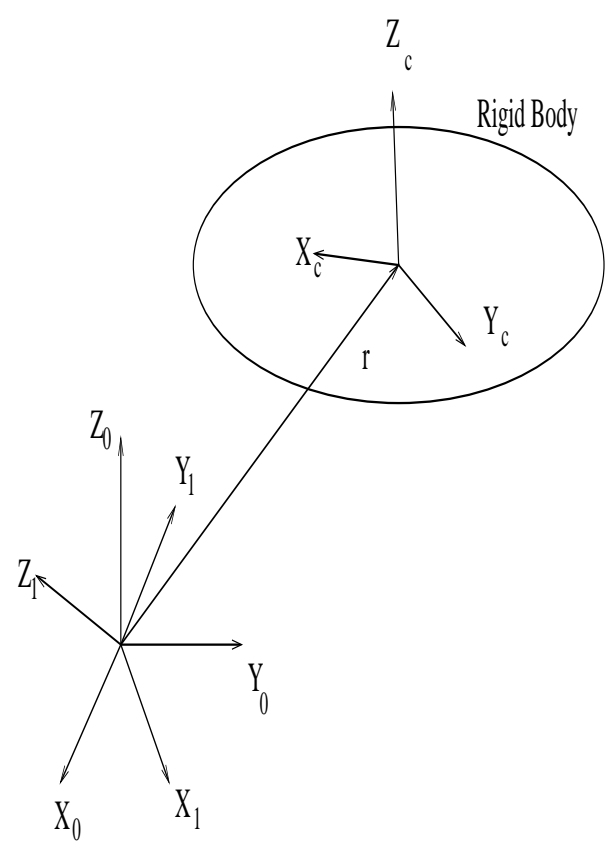

Fig. 2. A General Rigid Body showing Inertial Frame $X_{0}, Y_{0}, Z_{0}$, rotated from $X_{1}, Y_{1}, Z_{1}$ and Body Frame $X_{c}, Y_{c}, Z_{c}$

small) duration of the impact event and by considering the resulting impulsive forces. The velocity change is therefore determined by considering the impulse/momentum equations

$$
\left.\frac{\partial \mathcal{L}}{\partial \dot{q}}\right|_{t_{-}} ^{t_{+}}=W_{t_{-}}^{t_{+}}
$$

where $W_{t_{-}}^{t_{+}}$represents the impulse over $\left[t_{-}, t_{+}\right]$. Since

$$
\frac{\partial \mathcal{L}}{\partial \dot{q}}=\frac{\partial \mathcal{K}}{\partial \dot{q}}
$$

the quantity $\partial \mathcal{L} / \partial \dot{q}$ is invariant. Furthermore, the impulse $W_{t-}^{t+}$ is known to be linear combination of force one-forms $F_{i}$. These one-forms are the differentials of invariant functions describing the impact condition. Thus, these one-forms and the impulse $W_{t-}^{t+}$ are invariant. Therefore we have

Corollary 3.2. The velocity change due to the impact of the swing leg and ground is invariant under the slope changing action.

\section{CONTROLLED SYMMETRIES AND GRAVITY COMPENSATION}

We can use the above invariance properties to derive a control law that enforces invariance of solutions in the presence of gravity. We consider the controlled walking machine described by the impact dynamics and by the differential equation

$$
\frac{d}{d t} \frac{\partial \mathcal{L}}{\partial \dot{q}}-\frac{\partial \mathcal{L}}{\partial q}=B u
$$

where $B$ is a full rank matrix. Our main result can now be expressed as
Theorem 4.1. Let $\eta:[0, T] \rightarrow Q$ be a solution trajectory to equation (7) at $u=0$, Let $A \in \mathrm{SO}(3)$ and define

$$
\begin{aligned}
u & =B^{-1} \frac{\partial}{\partial q}\left(\mathcal{V}-\mathcal{V} \circ \Phi_{A}\right) \\
& =B^{-1} \frac{\partial}{\partial q}\left(\mathcal{V}(q)-\mathcal{V}\left(\Phi_{A}(q)\right)\right) .
\end{aligned}
$$

Then $\Phi_{A} \circ \eta:[0, T] \rightarrow Q ; t \mapsto \Phi_{A}(\eta(t))$ is a solution trajectory for the controlled walking machine (7).

Proof: Substituting the control law (8) into (7) and using invariance of the kinetic energy under the group action we have

$$
\frac{d}{d t} \frac{\partial \mathcal{L}_{A}}{\partial \dot{q}}-\frac{\partial \mathcal{L}_{A}}{\partial q}=0
$$

where $\mathcal{L}_{A}(q, \dot{q})=\mathcal{L}\left(\Phi_{A}(q), T_{q} \Phi_{A}(\dot{q})\right)$. Thus, if $\eta$ is a solution of (7) with $u=0, \Phi_{A} \circ \eta$ is a solution of $(9)$.

In particular, Theorem 4.1 together with Corollary 3.2 tells us that any limit cycles that exist for the passive walker for one ground slope can be reproduced by the active control law (8) for any other ground slope. Also, if $\left(q_{0}, \dot{q}_{0}\right)$ lies in the basin of attraction of the passive limit cycle, then $\left(\Phi_{A}\left(q_{0}\right), T_{q_{0}} \Phi_{A}\left(\dot{q}_{0}\right)\right)$ lies in the basin of attraction of the closed loop system. Thus, we are able to determine the appropriate initial conditions on any slope given one initial condition that leads to a passive gait on one particular slope.

\section{PASSIVITY BASED CONTROL}

The relationship with Passivity-Based Nonlinear Control is the following. Define the energy function $E_{\phi_{A}}$ as

$$
E_{\phi_{A}}(q, \dot{q})=\mathcal{K}(q, \dot{q})+\left(\mathcal{V} \circ \Phi_{A}\right)(q)
$$

where $\mathcal{K}, \mathcal{V}$ are the kinetic and potential energies of the biped. Then a simple calculation shows that, along trajectories of the system

$$
\dot{E}_{\phi_{A}}=\dot{q}^{T}\left(B U-\frac{\partial \mathcal{V}}{\partial q}+\frac{\partial\left(\mathcal{V} \circ \Phi_{A}\right)}{\partial q}\right)
$$

Define the Storage Function $\mathcal{S}$ as

$$
\mathcal{S}=\frac{1}{2}\left(E_{\phi_{A}}-E_{\mathrm{ref}}\right)^{2}
$$

where $E_{\text {ref }}$ is the (constant) energy of the biped along the limit cycle trajectory of the system (9). Note that $\mathcal{S}$ is identically zero on the limit cycle trajectory and non-negative away from the limit cycle. A simple calculation shows that 


$$
\dot{\mathcal{S}}=\left(E_{\phi_{A}}-E_{\mathrm{r} e f}\right) \dot{E}_{\phi_{A}}
$$

This suggests the following extension of Equation (8).

$$
u=B^{-1}\left(\frac{\partial \mathcal{V}}{\partial q}-\frac{\partial \mathcal{V} \circ \Phi_{A}}{\partial q}+\bar{u}\right)
$$

where $\bar{u}$ is an additional control input yet to be determined. Then it follows that

$$
\dot{\mathcal{S}}=\left(E_{\phi_{A}}-E_{\mathrm{r} e f}\right) \dot{q}^{T} \bar{u}
$$

Thus, if we choose the additional control $\bar{u}$ according to

$$
\bar{u}=-\left(E_{\phi_{A}}-E_{\mathrm{r} e f}\right) \dot{q}
$$

we have

$$
\dot{\mathcal{S}}=-\bar{u}^{2} \leq 0
$$

It can be shown using LaSalle's Invariance Principle (Khalil [1995]) that the manifold defined by

$$
\{\dot{q}=0\} \cap\left\{E_{\phi_{A}}=E_{\text {ref }}\right\}
$$

is attractive for the closed loop system over a single step. Indeed, for a system without impacts, all trajectories of the system would converge to this manifold asymptotically. Since impacts occur at finite intervals, LaSalle's Theorem cannot be used to predict the global behavior of the system. However, it seems intuitive that the basin of attraction of the stable limit cycle can be enlarged with the additional control term, baru. This is verified by simulations in the next section for the case of the compass gait biped.

\section{EXAMPLE}

Consider the compass gait biped from Goswami et al. [1997] shown below, The compass gait biped is equivalent to a double pendulum with point masses $m_{H}$ and $m$ concentrated at the hip and legs. The configuration of the compass gait is determined by the support angle, $\theta_{s}$, and nonsupport angle, $\theta_{n s}$. The dynamic equations, from Goswami, et. al. (1997), are

$$
M(q) \ddot{q}+C(q, \dot{q}) \dot{q}+g(q)=B u
$$

where $q=\left[\begin{array}{l}q_{1} \\ q_{2}\end{array}\right]=\left[\begin{array}{c}\theta_{n s} \\ \theta_{s}\end{array}\right] ; S=\left[\begin{array}{rr}-1 & 0 \\ 1 & 1\end{array}\right]$ and $u=\left[\begin{array}{l}u_{H} \\ u_{s}\end{array}\right]$.

The vector $u$ represents independent torques at the hip and ankle, which are assumed to be identically zero in the case of the passive biped.

The matrices $M(q), C(q, \dot{q})$, and vector $g(q)$ are given as,

$$
\begin{aligned}
& M(q)=\left[\begin{array}{cc}
m b^{2} & -m \ell b \cos \left(\theta_{s}-\theta_{n s}\right) \\
-m \ell b \cos \left(\theta_{s}-\theta_{n s}\right) & \left(m_{H}+m\right) \ell^{2}+m a^{2}
\end{array}\right] \\
& C(q, \dot{q})=\left[\begin{array}{cc}
0 & m \ell b \sin \left(\theta_{s}-\theta_{n s}\right) \dot{\theta}_{s} \\
m \ell b \sin \left(\theta_{n s}-\theta_{s}\right) \dot{\theta}_{n s} & 0
\end{array}\right] \\
& g(q)=\left[\begin{array}{c}
m b \sin \left(\theta_{n s}\right) \\
-\left(m_{H} \ell+m a+m \ell\right) \sin \left(\theta_{s}\right)
\end{array}\right],
\end{aligned}
$$

where $\ell=a+b$.

In terms of our convention for assigning coordinates, we may take $q_{1}=\theta_{s}$ the support angle and $r=\theta_{s}-\theta_{n s}$, the relative angle between the links (hip angle). Thus we see that the inertia matrix, and hence the Coriolis and centrifugal terms are independent of $q_{1}$.

Figure 4 shows a passive limit cycle for this system corresponding to a ground slope $\phi=3 \mathrm{deg}$. For

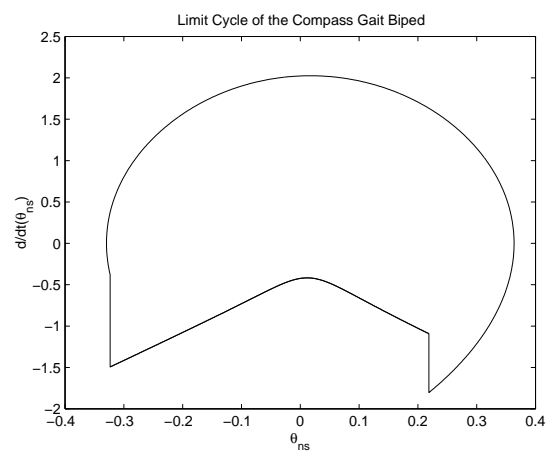

Fig. 3. Limit Cycle $\left(\theta_{n s}, \dot{\theta}_{n s}\right)$ for a Three Degree Slope

any ground slope, $\delta$, define $\beta=\phi-\delta$ and let $\mathcal{V}_{\beta}(q)=\mathcal{V}(q+\beta)$ and $g_{\beta}(q)=\frac{\partial \mathcal{V}(q+\beta)^{T}}{\partial q}$

Then we apply the feedback control law

$$
\begin{aligned}
u & =B^{-1}\left(\frac{\partial \mathcal{V}}{\partial q}-\frac{\partial \mathcal{V}_{\beta}}{\partial q}+\bar{u}\right) \\
& =B^{-1}\left(g(q)-g_{\beta}(q)+\bar{u}\right)
\end{aligned}
$$

where $\bar{u}$ is defined according to (16). Theorem (4.1) then implies that there is a stable limit cycle corresponding to the slope $\delta$ with $\bar{u}=0$. The additional term $\bar{u}$ vanishes on the limit cycle and its inclusion is intended to increase the basin of attraction and rate of convergence to the limit cycle. We illustrate this below via simulation.

Figures 5 and 6 show the effect of the added term $\bar{u}$. In the first plot $\bar{u}=0$ and the initial condition is chosen outside the basin of attraction of the limit cycle. The second plot shows that this same initial condition is now in the basin of attraction of the limit cycle with the addition of $\bar{u}$. 


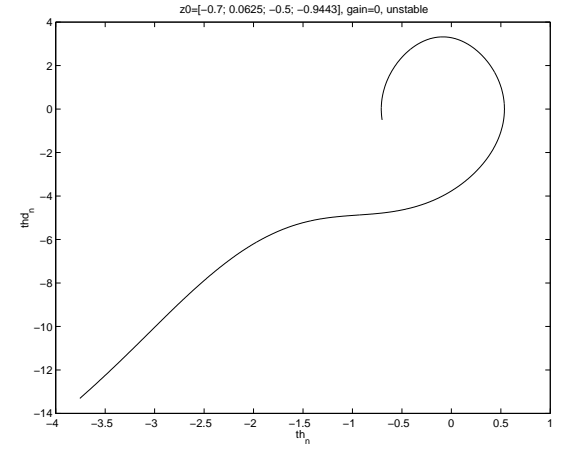

Fig. 4. Unstable response - initial condition is outside the basin of attraction

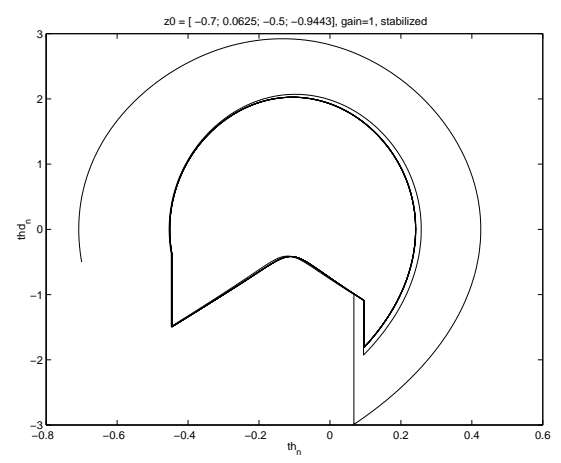

Fig. 5. Stable response using passivity based control $(k=1)$

\section{CONCLUSIONS AND FUTURE WORK}

The results in the paper have potential applications in developing energy efficient walking gaits for walking robots. Clearly, the passive limit cycle is energy optimal in that it requires no energy from external actuators. As the ground slope varies, the passivity based control law derived here is sub-optimal but may be used either as an initial guess in a search for an energy optimal control law or in lieu of one in case the search for the energy optimal control is prohibitively expensive.

\section{ACKNOWLEDGEMENTS}

This research was partially supported by NSF Grants ECE-9812591 and CSM-0100162. The authors would like to thank Gagandeep Bhatia and Ian Hiskens for help with the simulation results.

\section{References}

S. H. Collins, M. Wisse, and A. Ruina. A threedimensional passive-dynamic walking robot with two legs and knees. International Journal of Robotics Research, 20(7):607-15, 2001.

M. Garcia, A. Chatterjee, A. Ruina, and M. Coleman. The simplest walking model: Stability, complexity, and scaling. ASME Journal on Biomechanical Engineering, 120(2):281-288, 1998.

A. Goswami, B. Espiau, and A. Keramane. Limit cycles in a passive compass gait biped and passivity-mimicking control laws. Autonomous Robots, 4(3):273-86, 1997.

A. Goswami, B. Thuilot, and B. Espiau. A study of the passive gait of a compass-like biped robot: Symmetry and chaos. International Journal of Robotics Research, 17(12):1282-301, 1998.

Y. Hurmuzlu and D. Moskowitz. The role of impact in the stability of bipedal locomotion. Dynamics and Stability of Systems, 1(3):217234, 1986.

H. K. Khalil. Nonlinear Systems. Prentice Hall, Englewood Cliffs, NJ, 2 edition, 1995. ISBN 0132280248.

A. D. Kuo. Stabilization of lateral motion in passive dynamic walking. International Journal of Robotics Research, 18(9):917-30, 1999.

T. McGeer. Passive dynamic walking. International Journal of Robotics Research, 9(2):62-82, 1990.

T. A. McMahon. Muscles, reflexes, and locomotion. Princeton University Press, Princeton, NJ, 1984. ISBN 0691083223.

H. Ohta, M. Yamakita, and K. Furuta. From passive to active dynamic walking. In IEEE Conf. on Decision and Control, pages 38833885, Phoenix, AZ, December 1999.

P. J. Olver. Application of Lie Groups to Differential Equations, volume 107 of GTM. Springer Verlag, New York, 1993.

M. Spivak. A comprehensive introduction to differential geometry. Publish or Perish, Inc., Berkeley, CA, 2 edition, 1979. ISBN 0914098799.

M. W. Spong. Bipedal locomotion, robot gymnastics, and robot air hockey: A rapprochement. In TITech COE/Super Mechano-Systems Workshop, pages 34-41, Tokyo, Japan, February 1999a.

M. W. Spong. Passivity based control of the compass gait biped. In IFAC World Congress, volume 3, pages 19-23, Beijing, China, July 1999b.

M. W. Spong and M. Vidyasagar. Robot Dynamics and Control. John Wiley, New York, 1989. ISBN 047161243X.

S. Suzuki, K. Furuta, Y. Pan, and S. Hatakeyama. Biped walking robot control with passive walker model by new vsc servo. In American Control Conference, pages 107-112, Arlington, VA, June 2001. 